\title{
Diseño, fabricación y pruebas iniciales de una endoprótesis coronaria líberadora de fármaco
}

\author{
Arturo Abundes-Velasco, ${ }^{1}$ Jorge Padilla-Ibarra, ${ }^{2}$ Gian M. Jiménez-Rodríguez, ${ }^{2}$ Julio I. Farjat-Pasos, ${ }^{2}$ \\ Eduardo A. Arias-Sánchez, ${ }^{2}$ Félix Damas-de los Santos, ${ }^{2}$ Marco A. Martínez-Ríos, ${ }^{2}$ \\ Francisco J. Molina-Méndez, ${ }^{3}$ Tomás E. Sánchez-Pérez, ${ }^{1}$ Marco M. Arai-Ito, ${ }^{1}$ \\ Sebastián Aceves-Díaz González, ${ }^{1}$ David A. Rodríguez-Parra, ${ }^{1}$ Alberto Aranda-Fraustro, ${ }^{4}$ \\ Felipe A. Masso-Rojas, ${ }^{5}$ Ramsés Galaz-Méndez ${ }^{6}$ y Marco A. Peña-Duque ${ }^{1 *}$ \\ 'Instituto Nacional de Cardiología "Ignacio Chávez", Desarrollo e Innovación Tecnológica; 'Instituto Nacional de Cardiología "Ignacio Chávez", \\ Cardiología Intervencionista; ${ }^{3}$ nstituto Nacional de Cardiología "Ignacio Chávez", Anestesiología Cardiovascular; ${ }^{4}$ nstituto Nacional de Cardiología \\ "Ignacio Chávez", Patología; "Instituto Nacional de Cardiología "Ignacio Chávez, Fisiología; 'Instituto Tecnológico y de Estudios Superiores de \\ Monterrey Campus Ciudad de México, Ingeniería Biomédica. Ciudad de México, México
}

\section{Resumen}

Introducción: En el Instituto Nacional de Cardiología de México se desarrolla una endoprótesis (stent) coronaria liberadora de fármacos para el tratamiento de la cardiopatía isquémica. Objetivo: Establecer el mejor modelo animal para las pruebas, mostrar los avances en el prototipo del stent liberador de fármacos, evaluar la actividad antiproliferativa de dos fármacos y los resultados histológicos. Método: Se realizaron cultivos de células de músculo liso para evaluar las propiedades antiproliferativas de sirolimus y paclitaxel. Los fármacos fueron encapsulados en el interior de la matriz polimérica de los stents. Se emplearon conejos y cerdos como modelos animales. Resultados: Sirolimus y paclitaxel mostraron efecto inhibitorio, mayor en el segundo. La espectroscopia infrarroja y la microscopia óptica y electrónica mostraron que la capa del polímero con el fármaco se adhería adecuadamente al stent. A las cuatro semanas de seguimiento, ambos modelos animales mostraron evolución clínica satisfactoria y adecuada respuesta histológica, si bien el modelo porcino resultó más conveniente para protocolos futuros. Conclusiones: Las pruebas preliminares del stent liberador de fármaco brindó bases para desarrollar el protocolo con un número adecuado en cerdos y con seguimiento clínico angiográfico e histopatológico a tres meses.

PALABRAS CLAVE: Endoprótesis coronaria liberadora de fármaco. Sirolimus. Stent coronario.

\section{Design, manufacture and initial tests of a drug-eluting coronary stent}

\section{Abstract}

Introduction: A drug-eluting coronary stent is being developed at the National Institute of Cardiology of Mexico for the treatment of ischemic heart disease. Objective: To establish the best animal model for the tests, to show the advances in the drug-eluting stent prototype, to assess two drugs' antiproliferative activity and histological results. Method: Smooth muscle cell culture tests were performed in order to assess sirolimus and paclitaxel antiproliferative properties. The drugs were encapsulated inside the polymeric matrix of the stents. Rabbits and pigs were used as animal models. Results: Sirolimus and paclitaxel showed an inhibitory effect, which was higher for the latter. Infrared spectroscopy and light and optical microscopy showed that the drug/polymer layer properly adhered to the stent. At a four-week follow-up, both animal models showed satisfactory clinical evolution and adequate histological response, although the porcine model was shown to be more suitable for future

Correspondencia:

*Marco A. Peña-Duque

E-mail: marcopduque@gmail.com
Fecha de recepción: 09-12-2019

Fecha de aceptación: 17-03-2020

DOI: 10.24875/GMM.20005675
Gac Med Mex. 2020;156:279-285

Disponible en PubMed

www.gacetamedicademexico.com

0016-3813/@ 2020 Academia Nacional de Medicina de México, A.C. Publicado por Permanyer. Este es un artículo open access bajo la licencia CC BY-NC-ND (http://creativecommons.org/licenses/by-nc-nd/4.0/). 
protocols. Conclusions: Preliminary tests of the drug-eluting stent provided bases for the development of a study protocol with an adequate number of pigs and with clinical angiographic and histopathological three-month follow-up.

KEY WORDS: Drug-eluting coronary stent. Sirolimus. Coronary stent.

\section{Introducción}

Es incuestionable la importancia de las endoprótesis vasculares (stents) liberadoras de fármacos (SLF) en el manejo de la cardiopatía isquémica aguda o crónica. ${ }^{1-3}$ Los SLF de primera generación (Cypher ${ }^{\mathrm{TM}}$ y Taxus $^{\mathrm{TM}}$ ) ocasionaron un efecto espectacular en la práctica clínica, principalmente por la drástica reducción de la reestenosis: de $30 \%$ o más con los stents convencionales disminuyó a menos de $6 \%$.,5 Sin embargo, a largo plazo se mantenía el riesgo de trombosis $^{6}$ y se comenzó a reportar la presencia de neoateroesclerosis. ${ }^{7}$

Han sido importantes los avances en la tecnología de los actuales SLF de segunda generación: desde el material para su fabricación, el diseño, el recubrimiento y el tipo de polímero, hasta el tipo de fármaco antiproliferativo. ${ }^{8}$ Entre las mejoras destacan los numerosos metales y aleaciones que se han estudiado, los cuales brindan mayor fuerza radial o desplazamiento a los dispositivos. El soporte más utilizado en la actualidad es el fabricado con cromo-cobalto, el cual ha permitido disminuir el grosor de las paredes de las endoprótesis y, en consecuencia, la frecuencia de la reestenosis. Otra innovación son los polímeros bioabsorbibles que se aplican en la cara externa del dispositivo. ${ }^{9}$ En una publicación reciente en la que se comparó un SLF recubierta con polímero biodegradable que libera sirolimus versus un SLF recubierto por polímero no biodegradable que libera everolimus, a 12 meses de seguimiento se demostró que no existían diferencias. ${ }^{10}$

No cabe duda de que los progresos y mejoras continuarán y probablemente los mayores esfuerzos se encaminarán a lograr, además del efecto antiproliferativo, una endotelización más rápida y completa de la superficie tratada, para evitar no solo la reestenosis sino también la trombosis, el empleo de terapia antiplaquetaria dual a largo plazo y la neoateroesclerosis.

Desde hace varios años, en México se han realizado esfuerzos por desarrollar endoprótesis vasculares. $^{11-13}$ Desde 2014, en el Instituto Nacional de Cardiología se comenzó el desarrollo de un stent metálico desnudo (INC-01) con dos modelos, los cuales han sido sometidos a pruebas computacionales de elemento finito, así como pruebas mecánicas y biológicas, con resultados satisfactorios, ${ }^{14,15}$ por lo que se ha iniciado el protocolo en seres humanos.

Con la finalidad de continuar con este proyecto, se planeó desarrollar un SLF (INC D), con el mismo soporte del INC-01. En este reporte inicial se describen los avances, la evaluación antiproliferativa de varios fármacos, el establecimiento del modelo animal y la evaluación histológica de los resultados.

\section{Método}

En el Departamento de Fisiología del Instituto Nacional de Cardiología se efectuaron pruebas de las propiedades antiproliferativas en cultivo de células musculares lisas de dos fármacos: paclitaxel y sirolimus. El efecto de los medicamentos fue evaluado mediante citometría en cultivos de células musculares lisas, las cuales se tiñeron con CellTrace ${ }^{\mathrm{TM}}$, (Thermo Fisher); con el paclitaxel se emplearon dosis de 15, 50 y $100 \mathrm{nM}$ y en el sirolimus, dosis equimolares.

Los SLF fueron fabricados por la empresa GSE Biomedical y el Instituto Nacional de Cardiología, con el apoyo del Consejo Nacional de Ciencia y Tecnología. Se utilizaron los siguientes fármacos:

- Sirolimus (Vetranal ${ }^{\circledR}$, grado analítico) de Sigma-Aldrich.

- Paclitaxel ( $\geq 95 \%$, grado HPLC) de Sigma-Aldrich.

Se usó poliácido láctico, con una proporción de 20:80 de poliácido-L láctico:poliácido-D láctico. El estudio se llevó a cabo en 18 stents INC-01 de aleación cromo-cobalto.

El recubrimiento del polímero se colocó por pulverización a través de un actuador ultrasónico de $113 \mathrm{kHz}$ y $4-12 \mathrm{~V}$ a $4.5 \mathrm{~cm}$ del sustrato, con soluciones de concentración $1.0 \%$ w/v de poliácido láctico.

Los fármacos se integraron encapsulados en el interior de la matriz polimérica en una proporción de 20:80 de peso del fármaco y el polímero, respectivamente, siguiendo las concentraciones recomendadas en la literatura. ${ }^{16,17}$

De los 18 stents, seis fueron recubiertos con la preparación de sirolimus (INC D1) y seis con la preparación de paclitaxel (INC D2), manteniendo la 
cantidad recomendada de $150 \pm 10 \mu \mathrm{g}$ de los fármacos por stent. Todos los materiales fueron esterilizados en autoclave.

Se evaluó el recubrimiento de los stents mediante microscopia óptica digital y por espectroscopia infrarroja para confirmar la ausencia de interacción entre la matriz polimérica y el fármaco encapsulado; además, se realizó un ensayo de microscopia electrónica de transmisión para calcular el grosor del recubrimiento.

La preparación y montaje de los SLF se llevó a cabo en un área especialmente diseñada para montaje de dispositivos médicos, con ambiente controlado y en condiciones de esterilidad. Se adquirieron balones para angioplastia (genéricos): dos de $3.0 \mathrm{~mm}$ de diámetro $\times 20 \mathrm{~mm}$ de longitud y dos de $3.5 \mathrm{~mm}$ de diámetro $\times 20 \mathrm{~mm}$ de longitud, sobre los cuales se colocaron las endoprótesis mediante equipo especial (dispositivo de prensado); la película de polímero se protegió con papel teflón. Se aseguró la sujeción y centrado del stent en el balón. Se realizó la medición del perfil del stent montado mediante un calibrador vernier y mediante microscopia estereoscópica se revisó la superficie. Se montaron dos stents con paclitaxel sobre balones de 3.0 y $3.5 \mathrm{~mm}$ de diámetro, respectivamente. Se procedió de la misma forma con dos stents con sirolimus.

Se realizó empaquetado y sellado individual de los dispositivos, con la identificación del fármaco y el tamaño del balón. La esterilización se realizó mediante gas.

\section{Modelo lepórido}

Se emplearon dos conejos para cada una de las tres técnicas de acceso vascular que se probaron: disección carotídea, disección femoral y punción percutánea, las cuales resultaron de difícil ejecución, requirieron mucho tiempo y presentaron complicaciones locales. El acceso vascular se llevó a cabo sin complicaciones solo en un espécimen mediante punción percutánea, en el cual se implantó el stent INC D1 (sirolimus). Se trató de un conejo Nueva Zelanda (Oryctolagus cuniculus) macho de cuatro meses y $3.5 \mathrm{~kg}$, obtenido del Centro de Enseñanza, Investigación y Extensión en Producción Avícola de la Universidad Nacional Autónoma de México (UNAM), previa autorización del comité científico y de manejo de animales del Instituto Nacional de Cardiología. El conejo recibió aspirina y clopidogrel como premedicación y fue sedado una hora antes del procedimiento quirúrgico.
Se realizó punción de la arteria femoral izquierda mediante técnica de Seldinger y se colocó un catéter introductor de calibre $4 \mathrm{Fr}$. Se administraron 400 unidades de heparina. Se dirigió una guía coronaria de 0.014" hasta el arco aórtico y a continuación se desplazó el stent INC D1 de $3.5 \times 18 \mathrm{~mm}$, el cual se implantó en la aorta abdominal a 16 atmósferas, con una relación stent/aorta de 1.2/1 (ligeramente sobreexpandido). La adecuada expansión del stent fue corroborada mediante aortografía de control. El tiempo de fluoroscopia fue de 12 minutos y se utilizaron $10 \mathrm{~mL}$ de medio de contraste (Hexabrix ${ }^{\mathrm{TM}}$, Guerbet).

Durante cuatro semanas de seguimiento se administraron $50 \mathrm{mg} / \mathrm{día}$ de aspirina y $7.5 \mathrm{mg} / \mathrm{día}$ de clopidogrel, ambos por vía oral. Al finalizar el seguimiento se realizó eutanasia bajo sedación profunda con $1 \mathrm{mEq} / \mathrm{kg}$ de solución de cloruro de potasio. Posteriormente se realizó autopsia de corazón, corte de aorta torácica-abdominal y extracción manual de la endoprótesis vascular. Se llevaron a cabo cortes de $5 \mu \mathrm{m}$ de las muestras, las cuales fueron fijadas en formol a $10 \%$. El estudio histopatológico se realizó con tinciones de hematoxilina-eosina y de Masson y el análisis morfométrico del sitio tratado se efectuó en el Departamento de Patología del Instituto Nacional de Cardiología.

\section{Modelo porcino}

Se utilizó un solo espécimen, el cual resultó exitoso, un cerdo Yorkshire hembra de tres meses de edad y $46 \mathrm{~kg}$, obtenido del Centro de Enseñanza, Investigación y Extensión en Producción Porcina de la UNAM, previa autorización del comité científico y de manejo de animales del Instituto Nacional de Cardiología. Se administró premedicación con $100 \mathrm{mg}$ de aspirina y $300 \mathrm{mg}$ de clopidogrel.

Previa sedación, asepsia y antisepsia de la región femoral derecha y anestesia local con lidocaína simple a $2 \%$, se realizó punción de arteria femoral derecha; mediante técnica de Seldinger se colocó un catéter introductor femoral de calibre $6 \mathrm{Fr}$. Posteriormente, se introdujo guía teflonada de 0.035 " para continuar con el desplazamiento de un catéter guía tipo Judkins derecho de calibre $6 \mathrm{Fr}$. Se instaló una cánula en la arteria coronaria derecha, para introducir un filamento guía de 0.014" al segmento distal y avanzar el stent INC D2 (paclitaxel) de $3.5 \times 18 \mathrm{~mm}$, el cual se implantó en el segmento medio de la arteria coronaria derecha a una presión de 18 atmósferas, con una relación stent/ 


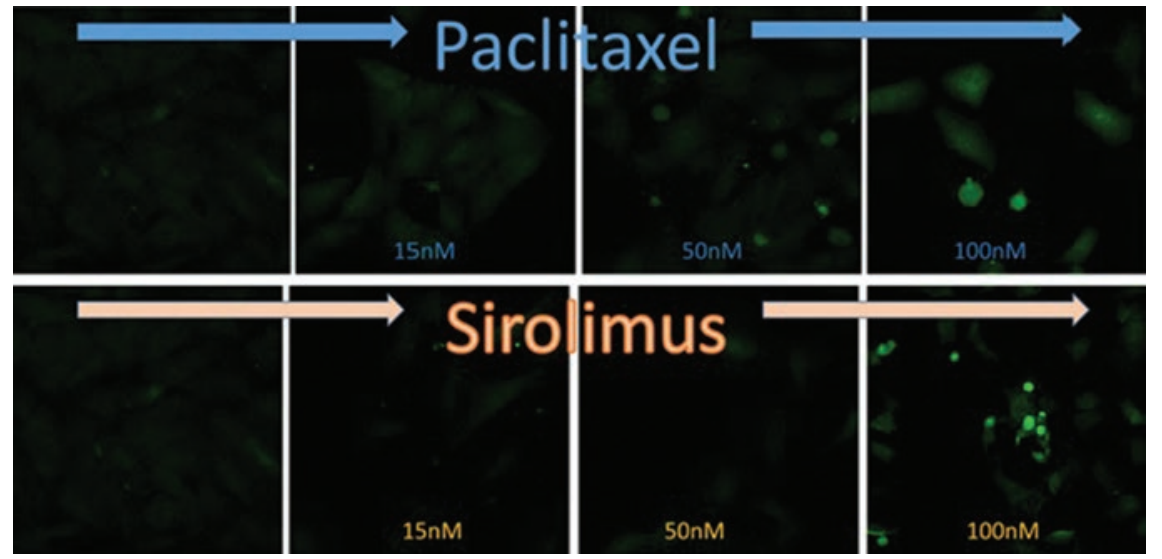

Figura 1. Tinción celular mediante método CellTrace: de izquierda a derecha se puede observar la disminución de la división celular con paclitaxel. La mayor intensidad demuestra que las células se dividieron menos veces; la retención del tinte indica la actividad antiproliferativa.

coronaria de 1.2/1. Posteriormente se insufló el balón para corroborar la adecuada expansión del stent. Se utilizaron $80 \mathrm{~mL}$ de medio de contraste (Hexabrix). El tiempo de fluoroscopia fue de 12 minutos. Se emplearon 7000 unidades de heparina no fraccionada.

Durante las cuatro semanas de seguimiento, se administraron $100 \mathrm{mg} /$ día de ácido acetilsalicílico y $75 \mathrm{mg} /$ día de clopidogrel, vía oral. Al final de este periodo se realizó eutanasia bajo sedación profunda con $1 \mathrm{mEq} / \mathrm{kg}$ de solución de cloruro de potasio, seguida de autopsia de corazón y disección de arterias coronarias, fijación con formol a $10 \%$, tratamiento con electrodeposición del stent INC D2 ${ }^{18}$ y cortes con microtomo de $5 \mu \mathrm{m}$. Para el estudio histopatológico se utilizaron tinciones de hematoxilina-eosina y de Masson. El análisis morfométrico de la arteria coronaria tratada se realizó en el Departamento de Patología del Instituto.

\section{Resultados}

\section{Del efecto antiproliferativo evaluado}

Mediante tinción CellTrace se identificó que el número de células resultó menor conforme aumentó la dosis del fármaco, pero la intensidad del colorante fue mayor, lo que sugiere menor división celular y, por lo tanto, mayor tinción del colorante, lo cual indica la actividad antiproliferativa de los dos medicamentos.

Con las mismas dosis de sirolimus se observó que si bien las células mostraban mayor intensidad del colorante, existió mayor número de células que en la evaluación de paclitaxel, cuyo efecto antiproliferativo al parecer es superior al del sirolimus a dosis equimolares (Figura 1).

\section{Del recubrimiento polímero/fármaco}

Se corroboró la integridad de la película del poliácido láctico de las endoprótesis mediante fotomicrografías.

Los SLF se sometieron a microscopia electrónica de transmisión. El recubrimiento polimérico con paclitaxel y sirolimus tuvo un grosor de 0.600 y $0.130 \mu \mathrm{m}$, respectivamente (Figura 2).

La ausencia de señales nuevas en la espectroscopia infrarroja indicó que no se formaron grupos funcionales nuevos como producto de una reacción entre la matriz polimérica y el fármaco encapsulado.

\section{Del modelo lepórido}

Se consideró que la intervención resultó exitosa. Mediante angiografía se confirmó diámetro luminal adecuado después del implante, desplazamiento normal del medio de contraste, sin evidencia de disección, fugas de material de contraste o trombo intravascular; además, se preservó la anatomía del sitio tratado y los vasos secundarios. No se presentaron complicaciones con el implante del stent INC D1 en la aorta abdominal de conejo ni eventos adversos.

Antes de este modelo se intentaron sin éxito dos implantes, el primero por disección quirúrgica carotídea y el segundo por disección inguinal de la femoral. 

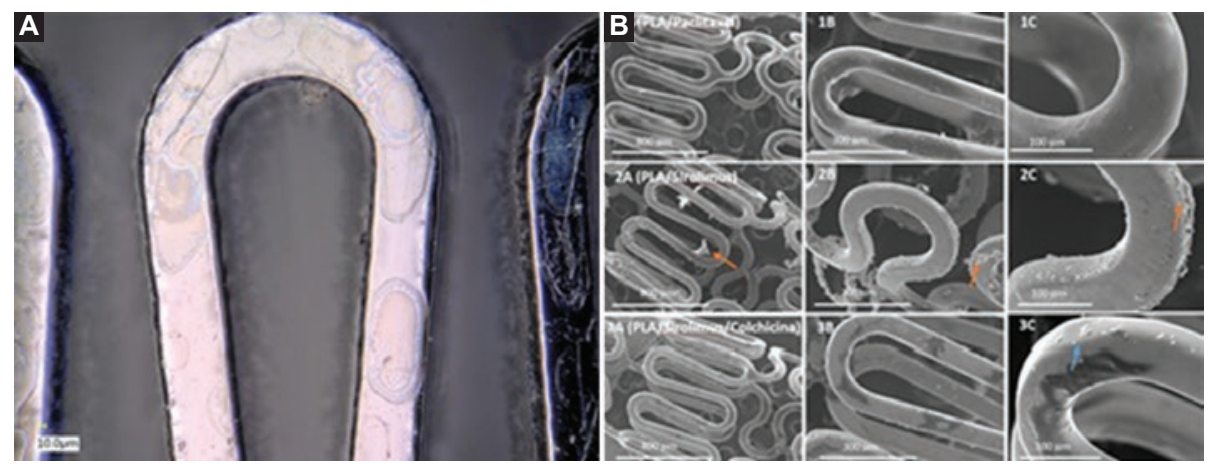

Figura 2. A) Microscopia electrónica del recubrimiento de polímero sobre la superficie de la endoprótesis vascular de cromo-cobalto. B) Microscopia electrónica de barrido en tres aumentos (75, 200 y 500x): $1 A, 1 B$ y $1 C$, polímero que libera paclitaxel; $2 A, 2 B$ y $2 C$, polímero que libera sirolimus.

\section{Del modelo porcino}

La intervención fue exitosa, sin complicaciones ni eventos adversos. Después del implante, por angiografía se corroboró expansión adecuada de la arteria, desplazamiento normal del medio de contraste, sin evidencia de disección, fuga de material de contraste o trombo intravascular; se preservó la anatomía del sitio tratado y los vasos secundarios. Este modelo resultó más adecuado y con mayor parecido a la anatomía y fisiología humanas.

\section{Evaluación técnica del implante del stent}

La implantación percutánea resultó satisfactoria en ambos modelos: la liberación, desplazamiento, implantación y expansión de los SLF fueron adecuadas, así como el retiro del catéter balón liberador y el análisis angiográfico. Además, fue posible preservar los vasos colaterales, con flujo sanguíneo final normal.

\section{Seguimiento y resultados histopatológicos}

Ambos modelos mostraron aumento ponderal adecuado durante el seguimiento de cuatro semanas, sin presencia de eventos adversos.

La aorta de conejo se disecó en forma longitudinal para la extracción del stent. No se observó proliferación neointimal ni datos de inflamación o hemorragia. El stent INC D1 mostró alta capacidad para inhibir la proliferación celular y la inflamación. El stent INC-D2 se extrajo mediante corte transversal de la arteria coronaria del cerdo. Los puntales (struts) de la endoprótesis se observaron de café oscuro dispuestos en forma oval o redonda en la zona de transición de la íntima y el músculo liso. Se evidenció acentuada proliferación de la íntima sobre la endoprótesis. Los puntales se sujetaron parcialmente a la capa muscular, ligeramente esclerosada, y se proyectaron a la íntima fibrosa, con escasos lípidos en forma de gotas o circunferencias. El stent INC D2 no mostró potencia para inhibir la proliferación neointimal con la concentración del fármaco utilizado en el modelo porcino (Figura 3).

\section{Discusión}

El gasto en investigación y desarrollo experimental es un indicador $y$, a la vez, un promotor del crecimiento económico de los diferentes países. Gran parte de la economía de los países desarrollados se basa en el conocimiento científico y tecnológico

En Estados Unidos se destina $2.79 \%$ del producto interno bruto a investigación y desarrollo tecnológico, el promedio en Latinoamérica es de $0.62 \%$ y en México se invierte $0.44 \% .^{19,20}$ Para acercarnos a cifras más competitivas, es indudable que en nuestro país se requieren esfuerzos enormes en los cuales participen el gobierno y la iniciativa privada.

Con apoyo de GSE Biomedical y el Consejo Nacional de Ciencia y Tecnología, en el Instituto Nacional de Cardiología "Ignacio Chávez", se inició el proyecto de un SLF (denominado INC D). Dado que se trata de tecnología de punta y que se requiere la selección adecuada de cada componente y recurso del proyecto, se estableció la ruta de desarrollo descrita en este manuscrito. Los resultados obtenidos son la base para elaborar el próximo proyecto de investigación: el protocolo animal con el stent INC D, con seguimiento de tres y seis meses para estudio final angiográfico e histopatológico.

Con base en esta exploración inicial concluimos que continuaremos con el soporte de cromo-cobalto, aleación que tiene una excelente fuerza radial, es 


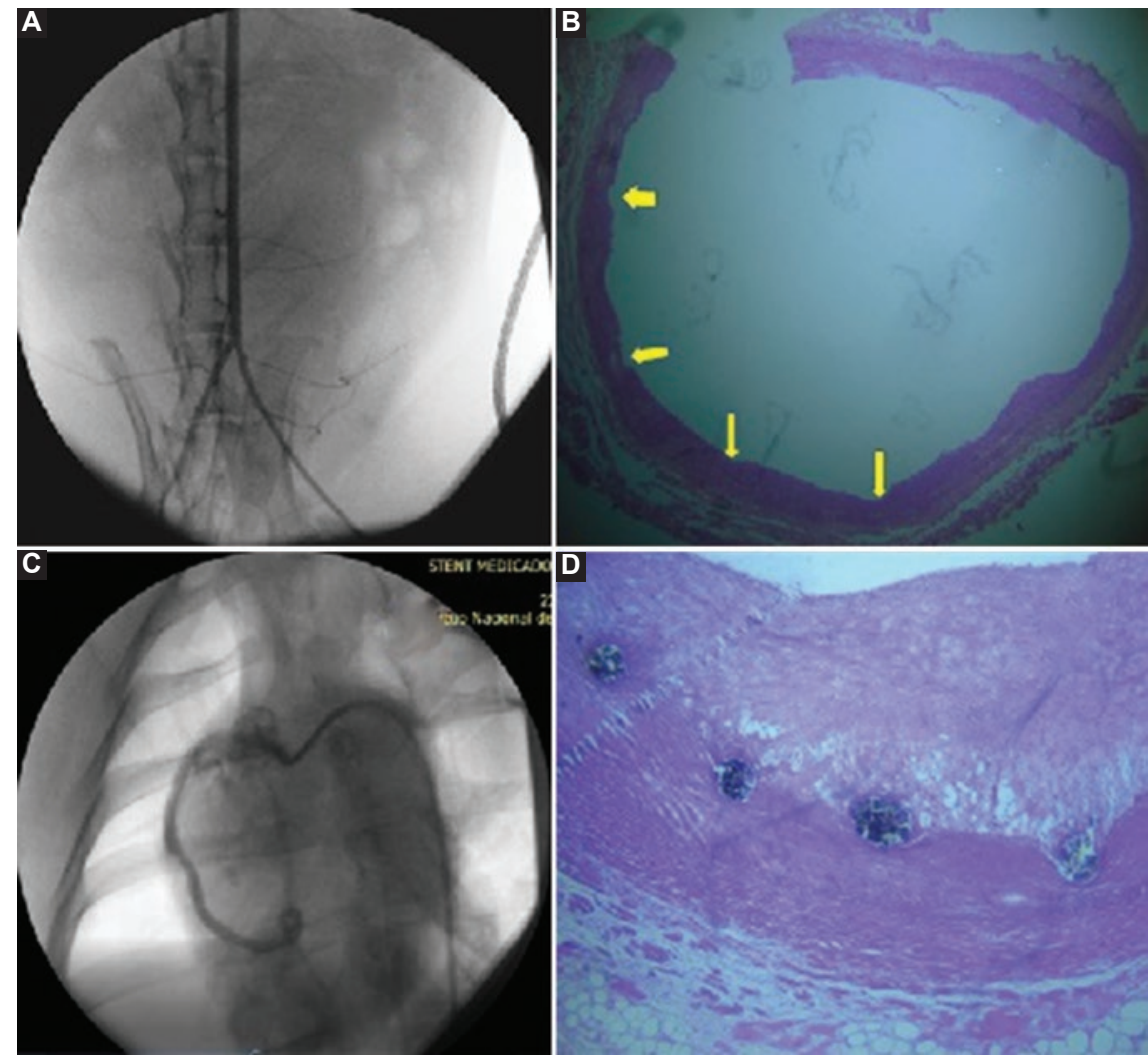

Figura 3. Angiografías e imágenes histopatológicas de los modelos animales. A y B) Modelo lepórido en el que se llevó a cabo acceso percutáneo femoral izquierdo. La angiografía muestra integridad vascular de la aorta con el stent INC-D1 (sirolimus) y en la fotomicrografía de la aorta del conejo se observan las muescas (flechas amarillas) que dejan los puntales del stent INC-D1 (sirolimus), sin proliferación neointimal. C y D) Modelo porcino. La imagen angiográfica se obtuvo después del implante del stent INC-D2 (paclitaxel) en el tercio medio de la coronaria del cerdo. En la fotomicrografía de la arteria coronaria derecha del cerdo en corte transversal con el stent INC-D2 (paclitaxel) se observan dos puntales que sujetan parcialmente la capa muscular discretamente esclerosada y se proyectan a la íntima fibrosa con escasos lípidos en forma de gotas o circunferencias (40x).

radiopaca y puede ser sometida a resonancia magnética de imagen, lo que permite su visualización mediante fluoroscopia convencional. ${ }^{21}$

El protocolo con el INC D se realizará con recubrimiento de polímero biodegradable (poliácido láctico) en la cara externa del dispositivo.

Algunos poliésteres termoplásticos como el poliácido glicólico (PGA), poliácido láctico-coglicólico (PLGA) y el poliácido láctico han demostrado ser materiales biodegradables adecuados para aplicaciones médicas. Debido a su grupo metilo, el poliácido láctico es más hidrofóbico que el PGA y el PLGA, por lo que es más adecuado para el encapsulamiento de sustancias con baja o nula solubilidad en agua, como el sirolimus. ${ }^{9}$ La biodegradación del poliácido láctico ocurre por hidrólisis, en la cual el ácido láctico liberado es eliminado por el cuerpo a través del ciclo de Krebs en forma de bióxido de carbono y agua, productos metabólicos no tóxicos. ${ }^{9}$

El recubrimiento solo de la cara externa del SLF tiene la finalidad de inhibir el crecimiento celular y la proliferación neointimal únicamente en la pared del vaso en contacto con los puntales del stent y, por otro lado, favorecer que la superficie luminal sea más fácilmente colonizada por endotelio sano. ${ }^{22}$

El fármaco antiproliferativo para el INC D será sirolimus, por su importante actividad inmunosupresora que bloquea la síntesis proteica y el ciclo celular y porque ha demostrado mayor efectividad contra la reestenosis. En la actualidad, los macrólidos inmunosupresores son los más aceptados para incorporarlos a las endoprótesis vasculares farmacoactivas. ${ }^{8}$

El modelo animal será el porcino, ya que existe mayor evidencia en la literatura internacional para la evaluación de los SLF en esta especie, técnicamente el procedimiento es más sencillo y con menor morbimortalidad durante el implante. Para compensar el inconveniente del rápido aumento de talla y peso de los animales durante el seguimiento, ${ }^{23,24}$ se sobredimensionará ligeramente el diámetro del stent en 
relación con el diámetro del segmento de la coronaria seleccionada. ${ }^{25}$

La evaluación histológica se llevará a cabo mediante electrodeposición modificada, ${ }^{18}$ porque se trata de un método más rápido y económico que la fijación con metacrilato; además, permite la conservación intacta del tejido.

\section{Conclusiones}

Se iniciará la fase de experimentación con un número adecuado de cerdos con base en la evaluación de los fármacos, la factibilidad para producir la endoprótesis vascular, el procedimiento quirúrgico y el espécimen más apropiado. Si los resultados son satisfactorios, se continuará con el protocolo clínico.

Se destaca la importancia del desarrollo tecnológico de dispositivos realizados en México, lo que permitirá la independencia y competitividad del país.

\section{Conflicto de intereses}

Los autores declaran no tener conflicto de interés alguno.

\section{Financiamiento}

Los autores no recibieron patrocinio para llevar a cabo este artículo. El proyecto que se describe se realizó con apoyo de la empresa GSE Biomedical y con recursos propios del Instituto Nacional de Cardiología.

\section{Responsabilidades éticas}

Protección de personas y animales. Los autores declaran que los procedimientos seguidos se llevaron a cabo conforme a las normas éticas del comité de experimentación humana responsable, la Asociación Médica Mundial y la Declaración de Helsinki.

Confidencialidad de los datos. Los autores declaran que en este artículo no aparecen datos de pacientes.

Derecho a la privacidad y consentimiento informado. Los autores declaran que en este artículo no aparecen datos de pacientes.

\section{Bibliografía}

1. Windecker S, Kolh P, Alfonso F, Collet JP, Cremer J, Falk V, et al. 2014 ESC/EACTS Guidelines on myocardial revascularization: The Task Force on Myocardial Revascularization of the European Society of Cardiology (ESC) and the European Association for Cardio-Thoracic Surgery (EACTS) developed with the special contribution of the European Association of Percutaneous Cardiovascular Interventions (EAPCI). Eur Heart J. 2014;35:2541-2619.
2. Levine GN, Bates ER, Blankenship JC, Bailey SR, Bittl JA, Cercek B, et al. $2015 \mathrm{ACC} / \mathrm{AHA} / \mathrm{SCAl}$ focused update on primary percutaneous coronary intervention for patients with ST-elevation myocardial infarction: An update of the $2011 \mathrm{ACCF} / \mathrm{AHA} / \mathrm{SCAl}$ guideline for percutaneous coronary intervention and the 2013 ACCF/AHA guideline for the management of ST-elevation myocardial infarction: A report of the American College of Cardiology/American Heart Association Task Force on Clinical Practice Guidelines and the Society for Cardiovascular Angiography and Interventions. Catheter Cardiovasc Interv. 2016;87:1001-1019.

3. Montalescot G, Sechtem U, Achenbach S, Andreotti F, Arden C, Budaj A, et al. 2013 ESC guidelines on the management of stable coronary artery disease: The Task Force on the management of stable coronary artery disease of the European Society of Cardiology. Eur Heart J. 2013;34:2949-3003.

4. Stone GW, Ellis SG, Colombo A, Grube E, Popma JJ, Uchida T, et al. Long-term safety and efficacy of paclitaxel-eluting stents final 5-year analysis from the TAXUS Clinical Trial Program. JACC Cardiovasc Interv. 2011:4:530-342.

5. Sousa JE, Costa MA, Abizaid A, Feres F, Seixas AC, Tanajura LF, et al. Four-year angiographic and intravascular ultrasound follow-up of patients treated with sirolimus-eluting stents. Circulation. 2005;111:2326-2329.

6. Stone GW, Moses JW, Ellis SG, Schofer J, Dawkins KD, Morice MC, et al. Safety and efficacy of sirolimus- and paclitaxel-eluting coronary stents. N Engl J Med. 2007;356:998-1008.

7. Ueda $Y$, Matsuo K, Nishimoto $Y$, Sugihara R, Hirata A, Nemoto T, et al. In-stent yellow plaque at 1 year after implantation is associated with future event of very late stent failure: The DESNOTE Study (detect the event of very late stent failure from the drug-eluting stent not well covered by neointima determined by angioscopy). JACC Cardiovasc Interv. 2015;8:814-821.

8. Lee $\mathrm{DH}$, de la Torre-Hernandez JM. The newest generation of drug-eluting stents and beyond. Eur Cardiol. 2018:13:54-59.

9. Parker T, Davé V, Falotico R. Polymers for drug eluting stents. Curr Pharm Des. 2010;16:3978-3988.

10. De Winter RJ, Katagiri Y, Asano T, Milewski KP, Lurz P, Buszman P, et al. A sirolimus-eluting bioabsorbable polymer-coated stent (MiStent) versus an everolimus-eluting durable polymer stent (Xience) after percutaneous coronary intervention (DESSOLVE III): A randomised, single-blind, multicentre, non-inferiority, phase 3 trial. Lancet. 2018:391:431-440.

11. Abundes AV, Quintana PG, Navarro RJ, Ledesma VM, Autrey CA, Solorio MS, et al. Estudio piloto preliminar en humanos con la prótesis endovascular (stent saq). Resultados inmediatos y a mediano plazo. Arch Cardiol Mex. 2000;70:377-383.

12. Abstracts: Oral and poster presentations. The Society for Cardiac Angiography and Interventions' 24 $4^{\text {th }}$ Annual Scientific Sessions, May 16-19, 2001. Westin Galleria Houston, Texas. Cathet Cardiovasc Intervent. 2001; $53: 97-122$

13. Abundes A, Rivera JJ, Arizmendi E, Farell J, Ledesma M, Montoya S. Immediate and long-term results of implantation of the new platinum coronary stent (atlas stent) in patients with coronary artery disease. Rev Esp Cardiol. 2002;55:1205-1208.

14. Abundes A, Romero JL, Sandoval JP, Galaz R, Ulacia P, Peña MA, et al. Diseño y fabricación de un stent coronario e inicio de pruebas en animales de experimentación. Rev Mex Cardiol. 2016;27.

15. Abundes A, Rodríguez E, Custodio P, Romero JL, Gala R, Ulacia P, et al. Experiencia fase animal del STENT coronario INC. Arch Cardiol Mex. 2017;87:79.

16. Waugh J, Wagstaff AJ. The paclitaxel (TAXUS)-eluting stent: A review of its use in the management of de novo coronary artery lesions. Am J Cardiovasc Drugs. 2004;4:257-268.

17. Vishnevetsky D, Patel P, Tijerino H, Gandhi PJ. Sirolimus-eluting Coronary Stent. Am J Health Syst Pharm. 2004:61:449-456.

18. Zúñiga-Ayala M, Juárez-Alvarado $O$, Aranda-Fraustro $A$, Velázquez-Espejel R, Becerra-Luna B, Peña-Duque M. Estudio histopatológico de arteria coronaria, tratada con stent metálico, posterior a la técnica de electrodeposición. Arch Cardiol Mex. 2017:87:81-82.

19. De la Peña L. Ciencia y tecnología en México, país dependiente. Ciencias. 1987; $10: 38-45$

20. Grupo Banco Mundial [Internet]. EE. UU.: Gasto en investigación y desarrollo (\% del PIB); 2020

21. Karanasiou GS, Papafaklis MI, Conway C, Michalis LK, Tzafriri R, Edelman ER, et al. Stents: Biomechanics, biomaterials, and insights from computational modeling. Ann Biomed Eng. 2017;45:853-872.

22. Kozuma K. Has the development of drug-eluting stents ended with limus-eluting stents? Circ J. 2018;82:330-331.

23. Suzuki $Y$, Yeung AC, Ikeno $F$. The representative porcine model for human cardiovascular disease. J Biomed Biotechnol. 2011;2011:195483.

24. Goodman SL. Sheep, pig, and human platelet-material interactions with model cardiovascular biomaterials. J Biomed Mater Res. 1999:45:240-250.

25. Schwartz RS, Edelman E, Virmani R, Carter A, Granada JF, Kaluza GL, et al. Drug-eluting stents in preclinical studies: updated consensus recommendations for preclinical evaluation. Circ Cardiovasc Interv. 2008:1:143-153. 